\title{
Avaliação de Sistemas de Informação: análise metodológica de vinte anos de publicações científicas
}

\author{
Fernando Skackauskas Dias \\ Universidade Federal de Minas gerais - PPGCI \\ fernandosd@ufmg.br
}

\begin{abstract}
Resumo. Através da revisão de artigos científicos, foi construído um quadro descritivo sobre as metodologias de Avaliação de Sistemas de Informação, delineando as principais características e estruturas. O interesse desta pesquisa é contribuir com um quadro atualizado sobre o tema de Avaliação de Sistemas de Informação. Para tal, foi realizada uma análise de artigos publicados em periódicos internacionais no período entre 1985 e 2005, totalizando 105 artigos. Foram utilizados os critérios de "Avaliação de Artigos de Pesquisa em Sistemas de Informação". Os resultados obtidos permitem demonstrar uma tendência das pesquisas para uma abordagem multidisciplinar e o alinhamento dos sistemas às estratégias corporativas.
\end{abstract}

\section{Introdução}

As pesquisas sobre sistemas de informação têm crescido enormemente nos últimos anos. Este fato se deve, entre outros, ao grande avanço tecnológico, à explosão informacional e à penetração dos sistemas nas mais diversas esferas da sociedade. Porém, como em toda área científica em emergência, as metodologias de pesquisa não conseguem acompanhar a velocidade dos acontecimentos. Isto pode interferir diretamente no desempenho dos pesquisadores e nos resultados das pesquisas.

Neste sentido, diversos especialistas têm desenvolvido metodologias de “Avaliação de Sistemas de Informação" com o objetivo de fornecer um arcabouço mais bem consolidado nesta área. Porém, pela forte característica multidisciplinar das investigações, estas abordagens metodológicas ainda são difusas e sem consenso (STRAUB, 1989). É comum a vários pesquisadores a constatação da ausência de critérios mais específicos que possam fornecer uma base sólida sobre as metodologias de avaliação de sistemas (GALLETTA \& LEDERER, 1989; GROVER et al, 1996 MYERS et al 1997; WOLSTENHOLME et al, 1999). Ou seja, como é possível averiguar os critérios utilizados nas avaliações? As avaliações seguem algum rigor metodológico? Existe algum instrumento que sirva como parâmetro para avaliar as avaliações? Como conseqüência da ausência de metodologias apropriadas para validar as avaliações em sistemas de informação, a área tem dificuldade em ser reconhecida cientificamente (STRAUB, 1989).

Para tal, foi realizada uma análise de artigos publicados em periódicos internacionais no período entre 1985 e 2005, totalizando 105 artigos. Para a construção da metodologia de pesquisa e interpretação dos artigos foram utilizados os princípios da “Análise do Conteúdo” de Bardin (1977), os critérios de “Avaliação de Artigos de 
Pesquisa em Sistemas de Informação” de Hoppen et al (1996) e os princípios de “Categorização de palavras-chave em Sistemas de Informação” de Barki et al (1993).

\section{Referencial teórico}

A temática de avaliação de sistemas surge em meados dos anos 1970 com novas abordagens, como as de Mason (1978) e Zmud (1979). Eles tomaram como base para a avaliação a teoria matemática da comunicação de Shannon e Weaver ${ }^{1}$, onde o objetivo era detectar os problemas de comunicação. Segundo estes autores, os problemas de comunicação se dão em três níveis. Primeiramente no nível técnico, tendo a acuidade como variável na transmissão dos símbolos. Em seguida se dá no nível semântico, onde se analisa a precisão da transmissão e, finalmente, no nível da eficácia, através do impacto no comportamento dos envolvidos. Estes parâmetros são utilizados como referencial na avaliação de sistemas e são fundamentais para a sua compreensão. Zmud (1979) define três categorias que devem ser consideradas em se avaliar o sucesso dos sistemas de informação que é o desempenho do usuário, uso do sistema e satisfação do usuário. As pesquisas demonstram na sua maioria, que medidas de eficácia dos sistemas devem ser vinculadas ao desempenho organizacional. Enquanto o valor de custo para implantação dos sistemas é de fácil mensuração, os benefícios são difíceis de avaliar e mensurar. Além disso, quanto mais próximo dos níveis mais altos da organização, ou seja, dos níveis estratégicos, é mais difícil avaliar os benefícios, pois a subjetividade, associada às tomadas de decisão é inevitável, tornando quase impossível avaliar a eficácia dos sistemas de informação e seu potencial estratégico. É de comum visão à maioria dos autores a falta de padronização das medidas e variáveis de avaliação de sistemas. Esta parece ser a maior barreira para avanços nesta área.

Com continuidade dos estudos, Ives e Olson (1984) elaboraram um dos primeiros trabalhos e definem duas classes de variáveis para a avaliação dos sistemas de informação: “Qualidade” e “Aceitação dos Sistemas de Informação” que compreendem as variáveis de usabilidade dos sistemas e impacto na operação das tarefas. Galletta e Lederer (1989), desenvolveram uma pesquisa onde verificaram a fidedignidade da avaliação de sistemas através da satisfação do usuário. O trabalho considera os motivos de satisfação como função da usabilidade e impacto na rotina de trabalho. Os autores ressaltam o cuidado na interpretação dos dados, pelo fato de que o instrumento de avaliação ser muito heterogêneo nos seus critérios. Delone e McLean (1993), definem a dimensão "Qualidade dos Sistemas de Informação", onde é considerado um conjunto de operações que recebem e processam dados a partir de regras. Este trabalho serviu de base para o trabalho de Myers, Kappelman e Prybutok (1997) que contribuem com as pesquisas de avaliação de sistemas ao questionarem e relacionarem os sucessos de avaliação através da eficácia e pertinência das variáveis utilizadas. Estes autores têm se debruçado com maior notoriedade sobre a temática e influenciaram diversos modelos posteriores, como o TTF - Task Technology Fit ${ }^{2}$ e o TAM - Technology Acceptance Model $^{3}$, largamente utilizados em avaliações de sistemas.

A literatura sobre avaliação de sistemas de informação é, conforme descrito pela maioria dos autores, bastante difusa e sem um consenso bem definido sobre técnicas e

\footnotetext{
${ }^{1}$ SHANNON, Claude Elwood \& WEAVER, Warren. The mathematical theory of communications, 1949

${ }^{2}$ GOODHUE, Anura \& THOMPSON, Ronald. Task-technology fit and individual performance. 1995

${ }^{3}$ DAVIS, Fred. Perceived usefulness, perceived ease use and user acceptance of information technology. 1989
} 
critérios metodológicos, necessitando de maiores investigações para melhor delimitar a temática como área de pesquisa científica.

\section{Metodologia}

Conforme é descrito por diversos pesquisadores em sistemas de informação (BARKI et al, 1993 e HOPPEN et al, 1996) não existe uma metodologia definitiva que possa servir como referência para pesquisas na área. Ou seja, é necessário cada pesquisador criar sua própria metodologia. Diante deste panorama, foi construído nesta pesquisa um arcabouço metodológico baseando em teorias já bem definidas e utilizadas. O método de análise dos artigos utiliza tipologias e classificações para modelar o quadro que se pretende construir, e métodos de interpretação de artigos científicos. A fonte que serviu de base para a organização das tipologias e das unidades de análise foi uma adaptação do trabalho desenvolvido por Barki et al (1993) e Hoppen et al (1996) onde foi construído um arcabouço para classificação e categorização de trabalhos científicos sobre sistemas de informação. Esta pesquisa tem um caráter qualitativo, portanto, para a seleção e interpretação dos artigos foram utilizados os princípios de Análise do Conteúdo (BARDIN, 1977).

Para se iniciar a pesquisa, a fonte de seleção dos artigos foi o portal CAPES ${ }^{4}$. Realizou-se uma busca na opção “Textos Completos" através da palavra-chave "Information System". A pesquisa retorna as editoras que publicam na temática referente ao assunto pesquisado. A seguir, em cada editora, realizou-se a segunda etapa de recuperação das fontes. No portal de cada editora, se fez a busca pelas palavraschave "assessment" e "evaluation". Ambas as palavras têm o sentido de "avaliação" na língua inglesa. É importante ressaltar que o processo de busca no portal de cada editora varia consideravelmente. Os mecanismos de busca de documentos permitem recuperações mais detalhadas, onde há uma maior precisão dos documentos recuperados e outros mecanismos menos detalhados, onde há um maior índice de revocação, ou seja, grande número de artigos pertinentes ao assunto. As palavras-chave "assessment" e "evaluation" foram pesquisadas no título dos artigos e no abstract, para permitir uma precisão maior de artigos que tratam sobre avaliação de sistemas.

Dentre os trabalhos publicados nos periódicos das editoras, foram selecionados aqueles que realizaram pesquisas efetivamente sobre avaliação de sistemas para atender o objetivo deste trabalho. Foi efetuada a busca pela entrada "Information System Assessment" e "Information System Evaluation". Dentre os trabalhos recuperados, o interesse da pesquisa se concentrou naqueles que tratam diretamente sobre a temática avaliação de sistemas de informação. Foram considerados tanto trabalhos empíricos quanto teóricos. Isto é interessante, considerando que esta pesquisa teve o objetivo de analisar o percurso da área, sendo este tema tratado tanto em trabalhos práticos que aplicam modelos inéditos para avaliação de sistemas, quanto à discussão de conceitos fundamentais. Os trabalhos teóricos permitem aprimorar a base desta pesquisa, pois demonstram características de tendências metodológicas. Houve um recorte temporal de 20 anos (1985 - 2005). Este recorte temporal concentra artigos que já estão disponíveis em arquivos eletrônicos, raros antes desta época.

Nos artigos analisados são considerados os principais elementos de avaliação, baseando-se no trabalho de Hoppen et al (1996). Segundo os autores, é essencial

${ }^{4}$ www.capes.periodicos.br 
questionar a pertinência do método de pesquisa, sua construção e uma adequação entre o método utilizado e o problema de pesquisa escolhido. Os elementos analisados consideram a questão da pesquisa que corresponde a várias dimensões do estudo. Na análise é preciso interrogar sobre os objetivos e sua pertinência. São considerados o rigor metodológico e o grau de controle adotado pelo autor da pesquisa. Neste elemento também são verificadas as bases teóricas utilizadas para fundamentar o estudo. $\mathrm{O}$ desenho de pesquisa é definido como a seqüência lógica que liga os dados da pesquisa à questão inicial e aos resultados. Deve-se identificar o objetivo do estudo e a estratégia utilizada para responder à pergunta fundamental da pesquisa. Neste elemento é importante verificar o tipo de estudo, se é um corte longitudinal ou transversal. O instrumento de medida e coleta dos dados é o elemento onde estão os conceitos relacionados com os procedimentos para o desenvolvimento dos instrumentos de medida, com os procedimentos de amostragem e os métodos de coleta de dados. A representatividade da amostra é uma característica fundamental para a interpretação de trabalhos, pois irá identificar a validade da representação junto ao universo existente. A validade do construto está diretamente relacionada à questão do que o estudo, através do instrumento de pesquisa, está realmente medindo. Para Peter (1981) o termo validade do construto refere-se à correspondência vertical entre um construto, que se encontra um nível conceitual inobservável, e uma medida que se pretende equivalente e que se encontra num nível operacional.

\section{4 resultados}

Os primeiros trabalhados tratam, mais especificamente, da avaliação de sistemas sob uma perspectiva tecnológica, ou seja, do uso e desempenho dos computadores. Estes trabalhos consideram como variável o número de transações efetuadas através da análise de $\log \mathrm{Ou}$ seja, é considerado como fator de sucesso ou fracasso as transações completadas ou que falharam. As pesquisas têm como objetivo validar e criticar modelos de avaliação de sistemas, onde aplicam o modelo de Nolan ${ }^{5}$, avaliando a evolução e uso da informática nas organizações. Estes trabalhos tinham como fundamentação justificar os investimentos tecnológicos e computacionais. Estes modelos mensuram o potencial dos sistemas através da relação entre investimento e aquisição em informática e uso dos computadores, substituindo processos manuais.

Muitos trabalhos avaliam a efetividade dos sistemas através da metodologia Servqual, já utilizada nos trabalhos de Delone e McLean em diferentes contextos. A metodologia Servqual se baseia na satisfação do usuário com base nos fundamentos de marketing e satisfação do consumidor. O objetivo é mensurar o intervalo existente entre as diversas expectativas criadas pelos usuários e o real desempenho dos sistemas, avaliando os motivos desta distorção.

Algumas abordagens propõem a avaliação de sistemas como forma integrada de diversas variáveis dependentes e independentes, considerando o ambiente, método, documentação e desempenho dos sistemas como fatores a serem avaliados. Estas perspectivas multivariáveis e em várias abordagens começam a surgir com mais freqüência a fim de aprimorar as técnicas de avaliação de sistemas. Um exemplo é a pesquisa de McBride (2003), que propõe a avaliação sob a abordagem da teoria do caos, em que os princípios são utilizados para explicar os fenômenos corporativos complexos, inclusive os sistemas de informação.

\footnotetext{
${ }^{5}$ NOLAN R. L. Managing the crisis in data processing. Harvard Business Review. 1979
} 
O modelo TAM (Technology Acceptance Model) é utilizado largamente como determinação da aceitação dos sistemas. Os autores agregam variáveis de usabilidade como longo prazo e curto prazo. A questão da usabilidade e do comportamento do usuário na busca pelas informações é tema recorrente em grande maioria dos artigos, em que retorna questões fundamentais sobre a usabilidade e comportamento psicológico do usuário, utilizando variáveis como tempo de resposta, compreensibilidade e efetividade da busca. Os trabalhos utilizam o conceito de "estado anômalo do conhecimento" de Belkin $^{6}$ (1988) como critério de busca de informação pelo usuário e o gap do conhecimento de Dervin ${ }^{7}$ (1984). Estas abordagens surgiram e começaram a ser utilizadas como abordagens multidimensionais.

O que se tem, na maioria das vezes, são avaliações que procuram integrar as dimensões usabilidade, qualidade dos sistemas e retorno esperado. São validados modelos de avaliação de sistemas através da adequação da informação e o impacto dos sistemas nas rotinas da organização. É interessante notar que a organização e disseminação do conhecimento são consideradas como variáveis dos sistemas, ou seja, até que ponto o sistema permite gerar conhecimento nas organizações (CHOO, 2003). Alguns autores desenvolvem suas pesquisas considerando as resistências encontradas pelos usuários dos sistemas como uma variável crítica para a difusão da informação e fracasso de diversos sistemas. Por outro lado, outros trabalhos são desenvolvidos para descrever como os usuários percebem os fatores ligados à satisfação dos sistemas em situações diferenciadas, que os autores chamam de "variações definicionais". Considera-se que usuários diferentes têm percepções diferentes sobre os mesmos sistemas. Isso acarreta níveis de avaliações diferenciados, dependendo do usuário, sobre a mesma realidade. Portanto, tais variações podem comprometer o nível de investimento em sistemas, como também a resposta às expectativas dos usuários.

As pesquisas sobre avaliação de sistemas retornam constantemente a atenção para os aspectos cognitivos dos usuários, na tentativa de mensurar o verdadeiro impacto na produtividade e no desempenho individual. Vários trabalhos focam na esfera psicológica / cognitiva dos desenvolvedores de sistemas e dos usuários. Segundo os autores, muita atenção é dada às questões externas aos sistemas, como características da informação, ambiente, software e hardware, em detrimento de aspectos mais relevantes. As pesquisas se concentram nas características cognitivas e dos conceitos e preconceitos e na relação destes fatores com o sucesso ou fracasso dos sistemas.

Outra grande parte das pesquisas tem como foco aspectos ligados ao desempenho organizacional e a relação dos sistemas com as estruturas corporativas. Considera-se como fator de avaliação de sistemas o papel exercido dos sistemas nas estratégias competitivas. Ou seja, o alinhamento existente entre os negócios e os sistemas de informação, suporte para decisões e posicionamento estratégico. Analisa-se o papel dos sistemas nas organizações e a relação entre os investimentos e o retorno. Tal preocupação começa a emergir fortemente nos trabalhos, pois as corporações investem altos montantes nos sistemas de informação sem perceber um retorno mais efetivo destes investimentos. Neste sentido, outras abordagens administrativas começam a surgir, como as que realizam a avaliação através dos auditores das empresas. As variáveis consideradas são a organização e disposição das informações para a auditoria, facilidade em fornecer a análise financeira e apoio estratégico. Outros trabalhos avaliam

\footnotetext{
${ }^{6}$ BELKIN N. J. Ask for information retrieval. Journal of Documentation. 1988.

${ }^{7}$ DERVIN, Brenda. The information needs of Californians. Institute of Governmental Affaire. 1984
} 
os recursos utilizados nos sistemas, como planejamento, segurança, integração de negócios e tecnológicos. Alguns pesquisadores avaliam os sistemas como suporte para as decisões financeiras, interferindo tanto no nível interno como no nível externo da empresa. Outras abordagens muito utilizadas são as metodologias para averiguar a confiabilidade nas informações, considerada crucial para a tomada de decisão. As variáveis são aplicadas através do número de erros ocorridos, número de acessos e opções disponíveis e não usadas.

Trabalhos com enfoques alternativos refletem sobre a ética nas avaliações de sistemas. O foco se dá no fator humano, no avaliador e nas características éticas, como a filosofia da avaliação, relações de poder, cultura, estilo gerencial e perfil do avaliador. Outras pesquisas têm uma perspectiva diferenciada, onde se avalia o sistema sob uma abordagem da semiótica, considerando como fatores de avaliação a estrutura, uso e conseqüência da informação. A análise se dá na relação entre o domínio de aplicação, complexidade, conseqüência social, aquisição, usabilidade de entrada e de saída, justificativa, relação dos sistemas com a realidade e representação dos sistemas na esfera estratégica. É sempre recorrente nas pesquisas as avaliações sobre a relação entre os investimentos em sistemas e o desempenho da empresa no ambiente macroeconômico. Ou seja, é possível medir a relação custo benefício de um sistema de informação? Como se relacionam os investimentos com o marketshare, valor de mercado, vendas e lucros? Neste sentido, algumas pesquisas avaliam o retorno dos investimentos em sistemas e consideram o tamanho da empresa como fator relevante na avaliação. Algumas pesquisas desenvolvem a investigação entre o alinhamento entre sistemas e as estratégias competitivas utilizando os conceitos de CMM. O CMM ${ }^{8}$ (Capability Maturity Model) é uma metodologia desenvolvida por pesquisadores da Carnegie Mellon para averiguar o nível de maturidade do desenvolvimento de software.

Os trabalhos mais recentes descrevem a avaliação de forma integrada baseando na melhoria contínua dos processos de avaliação do desempenho dos sistemas. Outros autores consideram como fatores determinantes do sucesso de sistemas a participação do usuário no desenvolvimento e sua inserção nas avaliações, como principal forma de mensurar o desempenho organizacional. Com o surgimento do ambiente web, as pesquisas começam a agregar variáveis específicas para estes sistemas, além de analisarem fatores de comportamento de busca. Diversos autores realizam a avaliação de sistemas em formatos hipertextuais, onde consideram características cognitivas e questionam se existe variação da motivação diante de textos hipertextuais ou lineares. Os trabalhos avaliam o sistema sob as dimensões da experiência do usuário, tipo de interface e estrutura de busca. Os autores consideram como fatores críticos para avaliação de hipertextos o tempo de espera pelo resultado de uma pesquisa, tempo de interpretação, quantidade da informação recebida, apoio à tomada de decisão e assimilação do conteúdo. O dimensionamento da tela, seqüência do diálogo, estrutura do documento, quantidade de nós, números de links, seqüência de hierarquia e número de referência são as variáveis analisadas. Outras pesquisas focam, também, características da Internet, considerando como métrica de avaliação o desempenho das máquinas de busca na web. As variáveis utilizadas são os números de páginas acessadas, retornadas, número de links, relevância da busca, escolhas da pesquisa, possibilidade de alteração, opções de busca, interface, qualidade dos resumos e uso de conectores lógicos.

${ }^{8}$ www.carnegiemellon.edu 
Questões como a relação dos sistemas e a integração com os processos administrativos têm sido exploradas por vários pesquisadores. Estes trabalhos avaliam o desempenho dos sistemas e seu poder de suporte aos aspectos gerenciais, como o EIS (Executive Information System), onde o foco principal é o impacto dos sistemas na produtividade, utilização dos recursos e auxílio nas tomadas de decisão. A aplicação da avaliação em sistemas que estruturam a cadeia produtiva é explorada por diversos pesquisadores, onde avaliam o desempenho de um sistema, como por exemplo, em um SCM - Supply Chain Management - e toda a estrutura informacional integrada da empresa Também o ERP - Enterprise Resource Planning - é investigado em diversos trabalhos. Os autores avaliam o ERP através da integração com os processos administrativos, a redução de custos e ganho de produtividade.

Por fim, algumas pesquisas têm como objetivo revisar fundamentos teóricos sobre o tema e a evolução dos conceitos fundamentais da área. Os autores abordam questões pertinentes aos sistemas de informação como forma de aprimorar os métodos de avaliação, pois, pode haver uma crise de conceitos nas avaliações, devido a sua intensa interdisciplinaridade e diversidade metodológica.

\section{Conclusões}

Os resultados obtidos permitem modelar um quadro que demonstra uma tendência das pesquisas para uma abordagem multidisciplinar, um interesse crescente nos aspectos cognitivos do usuário, foco na determinação dos atributos de qualidade da informação e no alinhamento dos sistemas de informação às estratégias corporativas. Existe uma forte característica de interligação com outras áreas do conhecimento, criando abordagens alternativas, como a aplicação do Balanced Scorecard, Gestão do Conhecimento, princípios da teoria do caos e da semiótica. A temática tem uma forte característica interdisciplinar, principalmente entre a Ciência da Computação, a Ciência da Informação e a Administração de Empresas. Constata-se uma tendência para pesquisas quantitativas e centradas no impacto dos sistemas na esfera organizacional e no desempenho produtivo, principalmente como forma de justificar os altos investimentos feitos em sistemas de informação. Como também é largamente analisado o impacto dos sistemas em todas as atividades corporativas, tanto no nível operacional, tático e estratégico. Com a forte característica de haver diversas áreas de conhecimento interessadas em analisar o mesmo fenômeno, o que se tem é um panorama amplo e complexo de metodologias, técnicas e variáveis, necessitando delimitar melhor os limites, características e escopo das pesquisas. Por fim, mesmo ainda em fase de definição teórica e metodológica, a avaliação de sistemas de informação tem surgido com novos formatos e características próprias nos grandes centros de pesquisa, já se formando um corpo sólido e bem delimitado de objeto de investigação.

\section{Referência bibliográfica}

BARDIN, Laurence. “Análise de Conteúdo”. Portugal: Edições 70, 1977.

BARKI, Henri; RIVARD, Suzanne; TALBOT, Jean. “A keyword classification scheme for IS research literature: an update”. MIS Quarterly. v. 17, n. 2, p. 209-225, 1993

CHOO, Chun Wei. “A Organização do conhecimento”. São Paulo: Editora Senac. 2003. 
DELONE, William \& McLEAN, Ephraim. "The DeLone and McLean Model of Information Systems Success: A Ten-Year Update”. Journal of Management Information Systems. Vol. 19, No. 4, pg. 9 - 39, 2003.

GALLETTA, Denis, LEDERER, F. "Some Cautions of the Measurement of User Information Satisfaction”. Decision Sciences, v. 20, pg. 419-438. 1989.

GROVER, Varun, JEONG, Seung Ryul, SEGARS, Albert. "Information System Effectiveness: the construct space and pattern of application”. Information \& Management, v. 31. p. 177-191, 1996.

HOPPEN, Norbeto; LAPOINTE, Liette; MOREAU, Eliane. "Um guia para a avaliação de artigos de pesquisa em Sistemas de Informação”. Revista de Administração. Ed 3, v. 2, n.2, 1996.

IVES, Blake \& OLSON, Margaret. "User Involvement and MIS Success: a review of research”. Management Science, v. 30, p. 586-603. 1984.

MASON, Ruchard O. "Measuring information output: a communication systems approach”. Information \& Management Review, v. 1, p. 219-234, 1978.

MCBRIDE, Neil "Chaos theory as a model for interpreting information systems in organizations”. Information Management, 2003.

MYERS, Rarry I. \& KAPPELMAN, Leon A. \& PRYBUTOK “A Comprehensive Model for Assessing the Quality and Productivity of the Information Systems Function. Toward a Contingency Theory for Information Systems Assessment”. Information Resources Management Journal, University of North Texas. 1997.

PETER, P. J. “Construct validity: a review of basic issues and marketing practices”. Journal of Marketing Research. P. 133-143. 1981.

STRAUB, D. W. "Validating instruments in MIS research”. MIS Quarterly, p. 147-169. 1989.

WOLSTENHOLME, Eric F. HENDERSON; GAVINE, Allan. "The evaluation of Management Information Systems. A dynamic and holistic approach”. London. Wiley Publisher, 1993.

ZMUD, Robert W. "Individual differences and MIS success: a review of the empirical literature”. Management Science, v. 25, p. 966-979, 1979. 